\title{
Un insolito caso di reazione anafilattica da farmaco: cenni clinici e riflessioni medico-legali
}

\author{
Valerio Cirfera ${ }^{1}$, Cosimo Prete ${ }^{2}$, Giancarlo Toma ${ }^{3}$, Paolo Vinci ${ }^{4}$ \\ Specialista in Dermatologia e Venereologia, Coordinatore nazionale del gruppo GIDEL - AIDA \\ (Associazione Italiana Dermatologi Ambulatoriali - Gruppo Italiano Dermatologia Legale) \\ 2 Avvocato, Foro di Lecce \\ 3 Specialista in Medicina Legale, Maglie (LE) \\ 4 Avvocato, patrocinante in Cassazione
}

\begin{abstract}
We report a case of a cutaneous ulcer in a 49 years-old patient with diabetes and hypertension. Penicillin in pownder for local use was prescribed. A severe anaphylactic reaction was observed. This case leads to some considerations concerning the off-label prescribing, that is to say the prescription of a registered medicine for a use that is not included in the product information.

Thus off-label prescribing in dermatology may sometimes be clinically appropriate, it may be associated with a number of clinical, safety and legal issues.

Off-label prescribing is acceptable if there is no suitable alternative and an appropriate process for informed consent is needed.

The practice of prescribing off-label drugs is common among dermatologists, many of whom had misperceptions about which are the approved indications of the drug and about the legal ramifications of off-label therapies. The authors suggest that understanding the principles of off-label prescribing in conjunction with the mechanisms of drug action in diseases may help clinicians in increasing the safety of their patients and in avoiding legal litigations.
\end{abstract}

Keywords: Adverse Drug Reaction, off-label penicillin, off-label dermatologic therapies, legal liability

An unusual case of anaphylactic reaction: clinical and legal aspects.

Pratica Medica \& Aspetti Legali 2007; 1(1): 7-12

\section{INTRODUZIONE}

Si riporta un caso pratico relativo ad una presunta responsabilità professionale correlata all'insorgenza di una grave reazione allergica sistemica, di tipo anafilattico, secondaria a somministrazione cutanea di antibiotico, nella fattispecie del gruppo delle penicilline naturali, somministrabili esclusivamente per via parenterale. L'interesse medicoclinico e medico-legale del caso è indubbio in virtù della sua eccezionalità, globalmente valutata, $\mathrm{e}$ dell'opportunità di esprimere delle considerazioni su problematiche di scottante attualità, quali il consenso informato, la corretta condotta operativa del medico e la conoscenza di norme e leggi che ad essa si riferiscono.

\section{DESCRIZIONE DEL CASO}

Paziente di 49 anni all'epoca dell'evento dannoso, di sesso femminile, diabetica e ipertesa da oltre 20 anni, richiedeva una visita medica specialistica per ulcere cutanee acrali del piede sinistro, con segni di flogosi concomitante, insorte da qualche mese e resistenti alla cura con i comuni trattamenti e presidi curativi, rispettivamente effettuati e adoperati con scarso successo dal medico di famiglia. Il Sanitario chiamato a consulto, dopo una prima indagine anamnestica e un successivo esame clinico, faceva diagnosi di ulcere infette delle dita del piede sinistro su base microangiopatica e consigliava terapia antibiotica per via generale da associare a disinfezione accurata delle ulcere per 
via locale. La paziente faceva presente che preferiva non assumere antibiotici per via sistemica in virtù di un malore, non meglio specificato, avuto circa 6 anni prima, in seguito alla somministrazione di antibiotici, mai più somministrati. La figlia precisava che l'antibiotico in causa, molto probabilmente, apparteneva alla classe delle cefalosporine, ma non poteva essere più precisa, dal momento che era passato del tempo dall'episodio. La paziente stessa aggiungeva che, oltre agli antibiotici, sin da bambina non tollerava i prodotti caseari, e talvolta, dopo che assumeva latte e derivati, notava l'insorgenza di prurito e orticaria diffusa a gran parte dell'ambito cutaneo.

Il medico specialista, alla luce di tali affermazioni, procedeva a prescrivere una toilette del piede con fisiologica e antibiotici solo ed esclusivamente per via locale e nella fattispecie, penicillina in fiale, per la sua nota efficacia battericida, raccomandando il seguente schema terapeutico: la polvere del flacone doveva essere applicata sulle ulcere cutanee due volte al giorno per due settimane, mentre il solvente non doveva essere utilizzato. Lo stesso Sanitario disponeva il controllo medico dopo 14 giorni.

Il mattino successivo, la figlia della paziente procedeva a toilette delle ulcere e subito dopo vi applicava la polvere, secondo le indicazioni mediche ricevute. In un lasso di tempo di circa 50 minuti, la paziente sviluppava intenso prurito su tutto l'ambito cutaneo, arrossamento e senso diffuso di calore, cardiopalmo, nonché lieve difficoltà respiratoria, via via più intensa nei minuti successivi al momento iniziale; trasportata d'urgenza al vicino presidio medico di pronto intervento, veniva immediatamente soccorsa, con difficoltà dei Sanitari nel reperire un accesso venoso, data la contemporanea caduta repentina dei valori pressori, causa diretta di uno stato sub-collassiale. Intanto anche lo stato di coscienza incominciava ad obnubilarsi e la dispnea ad accentuarsi.

Il personale infermieristico procedeva al lavaggio del piede e all'asportazione della polvere antibiotica visibile sul piede, mentre quello medico era costretto a isolare chirurgicamente un accesso venoso in sede sub-malleolare, quindi lo incannulava per l'opportuna terapia infusionale dettata dal caso specifico, integrata nelle manovre rianimatorie con somministrazione di ossigeno. Dopo circa mezz'ora di terapia continua, i valori pressori si ristabilivano a livelli compatibili con la vita, la situazione generale migliorava alquanto e l'evento veniva diagnosticato come "grave reazione avversa sistemica da farmaci” di tipo anafilattico, a causalità e patogenesi da determinare successivamente in opportuna sede. La paziente, trasferita in ambiente medico, riceveva le ulteriori terapie mediche per la stabilizzazione del quadro clinico e veniva sottoposta agli opportuni accertamenti del caso, tra cui il dosaggio delle IgE Prist e Rast per sospetta allergia alla penicillina; entrambi i titoli anticorpali risultavano maggiori rispetto ai valori normali standard di riferimento [1].

\section{GLI ASPETTI CLINICI}

Il caso, globalmente valutato, è senza dubbio insolito nella pratica clinica quotidiana, ma di notevole interesse scientifico per i molteplici punti di discussione analitica clinica che può evocare; non è scopo del lavoro soffermarsi su di essi in modo approfondito, avendo esso preminente finalità valutativa medico-legale e giuridica. Di certo le reazioni avverse alla penicillina somministrata per via parenterale e orale, comprese quelle gravi di tipo anafilattico e fatali, sono più che note in letteratura medica, anche da tempo, rappresentando senza ombra di dubbio esempi fra i più frequenti di ADR (Adverse Drug Reaction) [2-6].

Di contro, le reazioni locali, quelle sistemiche e soprattutto quelle anafilattiche da applicazione topica di penicillina naturale, sono meno frequenti rispetto a quelle suscitate dalla somministrazione per via generale; anche le relative pubblicazioni in letteratura [7] sono rare, e non potrebbe essere altrimenti, dal momento che le classiche penicilline iniettabili non sono previste per la somministrazione topica, che rappresenta di conseguenza una modalità d'uso off-label, ossia non autorizzata e, in linea di massima, da riservare a casi selezionati, per cui i dati statistici sono, al riguardo, limitati e talvolta datati [8]. Sono invece frequenti e note in dermatologia le reazioni avverse da antibiotici topici non penicillinici, a causa del loro largo uso consentito e condiviso in terapia; il riferimento è d'obbligo alle reazioni da sulfamidici, neomicina, mupirocina $[9,10]$, bacitracina ecc; in aggiunta si riportano alcuni riferimenti a casi di shock anafilattico alla bacitracina topica, reperibili anche in Rete $[11,12]$.

Nel caso in questione, le lesioni ulcerose dei piedi della paziente hanno favorito alquanto l'assorbimento sistemico del farmaco, a causa della vasodilatazione flogistica del microcircolo dermico e dell'esposizione dei tessuti lesi, privi di protezione cutanea. Da quanto fin qui esposto, si evince che la reazione avversa è stata di natura immunologica, verosimilmente di tipo 1 , mediata da $\lg E$ specifiche (Tabella I) dirette contro i determinanti antigenici della penicillina, noti e utilizzati nei test a finalità diagnostica in vitro e in vivo (Penicilloil-Poli-Lisina), modalità, quest'ultima, da valutare con attenzione, a causa della sua estrema rischiosità per i pazienti già sensibilizzati [13], come certamente la paziente protagonista di tale caso.

\section{GLI ASPETTI LEGALI}

Gli aspetti legali del caso in questione riguardano le condotte inappropriate o errate assunte dal Sanitario nell'indagine anamnestica e nella prestazio- 
ne d'opera professionale "de quo", che potrebbero essere interpertate come malpractice, concetto, questo, attualmente "di moda" nell'ampio panorama delle professioni sanitarie, a volte usato inopportunamente e ingiustamente ancor prima del giudizio di responsabilità [14], per definire una condotta sanitaria imperita, negligente, imprudente e non rispettosa delle norme, disposizioni e leggi statuite dall'ordinamento vigente in merito a "fatti sanitari" (art. 43 del Codice penale). La dimostrazione del nesso di causalità tra operato pregiudizievole e danno costituisce l'elemento fondamentale e probante della malpractice, momento imprescindibile per la successiva valutazione della responsabilità professionale, nei vari ambiti giuridici: civile a tutela del patrimonio dell'individuo e/o penale, a tutela della salute, bene supremo dell'uomo e di rango costituzionale.

\section{FASE ANAMNESTICA}

La fase anamnestica costituisce momento imprescindibile dell'attività sanitaria, atto medico esso stesso e premessa indispensabile per il risultato positivo del successivo iter diagnostico-terapeutico. Lanamnesi in caso di sospetta sensibilizzazione a farmaci o di pregressa reazione avversa ad essi è spesso l'unico strumento in mano del medico per orientare la sua condotta clinica e ben operare ai fini della prevenzione del danno alla persona, ovvero lo strumento più importante per la tutela della salute dell'assistito e della stessa professionalità del suo medico.

Il colloquio con il paziente deve essere puntuale sulla storia clinica precedente, estesa anche ai familiari, onde escludere stati (es. atopia) geneticamente favorenti le allergie; deve essere, altresì, adeguato alle capacità recettivo-cognitive del paziente e circoscritto al farmaco particolare, alla classe di farmaci di appartenenza e alle classi che possono essere interessate da allergia crociata con il farmaco prescritto (es. cefalosporine e penicilline). Utile l'ausilio e la collaborazione del medico di famiglia, specie se egli è a conoscenza della storia clinica del paziente, nonché l'aiuto dei parenti prossimi, se il paziente non è in grado di collaborare efficacemente. È altrettanto utile esaminare l'eventuale documentazione sanitaria in possesso dell'ammalato o conoscere eventuali luoghi di cura precedenti, al fine di "attingere" informazioni ausiliarie. Infine l'inchiesta anamnestica può giovarsi, per completezza, di un questionario specialistico redatto ad hoc o prelevato da lavori o pubblicazioni da enti scientifici riconosciuti ufficialmente $[15,16]$.

Nel caso descritto, l'indagine anamnestica è stata oggettivamente carente, o almeno così appare dall'esame della documentazione disponibile. Il dubbio su sospetti pregressi episodi di allergia o pseudoallergia avrebbe dovuto stimolare lo specialista a valutare meglio il rischio allergologico dell'assistita, tenendo conto dei parametri essenziali sopra menzionati. In aggiunta, purtroppo,

\begin{tabular}{|c|c|c|}
\hline Tipo & Meccanismo & Manifestazioni cliniche \\
\hline I & IgE mediato & $\begin{array}{l}\text { Anafilassi, orticaria, asma (allergie } \\
\text { in senso stretto) }\end{array}$ \\
\hline II & citotossico & $\begin{array}{l}\text { Anemia emolitica, } \\
\text { piastrinopenia,nefrite interstiziale }\end{array}$ \\
\hline III & immunocomplessi & Malattia da siero, vasculite \\
\hline IV & cellulomediato & Dermatite da contatto, rash \\
\hline
\end{tabular}

Tabella I. Reazioni immunopatologiche secondo Gell e Coombs

non vi sono prove che dimostrino l'effettuazione di un'anamnesi dettagliata, come il caso avrebbe richiesto; l'intolleranza non meglio specificata alle cefalosporine e ad alcuni alimenti che in teoria potrebbero essere stati contaminati da antibiotici avrebbe dovuto comportare un differimento della terapia antibiotica, da aggiornare e modulare in rapporto a una indispensabile valutazione dello stato di sensibilizzazione della paziente, in vitro ed eventualmente in vivo, stante anche il fatto che non ricorreva lo stato di urgenza medica e quindi della necessità di prescrivere e far effettuare a tutti i costi quel tipo di terapia. Ne discende che la condotta professionale del Sanitario non è esente da giudizi critici sia dal punto di vista medico che legale.

Si riportano, a tal proposito, le cause di giustificazione del medico, così come da egli stesso addotte:

- "ho consigliato la terapia a base di penicillina naturale per la sua elevata efficacia battericida in un contesto clinico a rischio di grave infezione sottocutanea e necrosi parcellare dei tessuti del piede in diabetica»;

- "ho preferito la via di somministrazione topica, anche se non prevista per le penicilline iniettabili, per la minore incidenza di effetti sistemici che tale via può garantire rispetto alla via generale, nell'ambito di un sospetto stato di sensibilizzazione»;

- «non ho provveduto all'approfondimento e accertamento diagnostico allergologico perché i test in vitro non danno risposte univoche e quelli in vivo sono pericolosi per la vita e poi perché poteva essere perso tempo prezioso".

\section{RIFLESSIONI}

A favore del Sanitario è da considerare che l'eventuale opzione per la somministrazione del farmaco per via generale, giustamente omessa, avrebbe potuto ipotecare uno shock anafilattico fatale; di fatto, nel giudizio globale di responsabilità è da tenere in debito conto tale evenienza. Tuttavia, se si rapporta tale tipo di ragionamento logico alla possibilità che ugualmente un trattamento topico, con una sostanza (penicillina) facilmente assorbibile nel circolo sistemico e gravata da reattività 


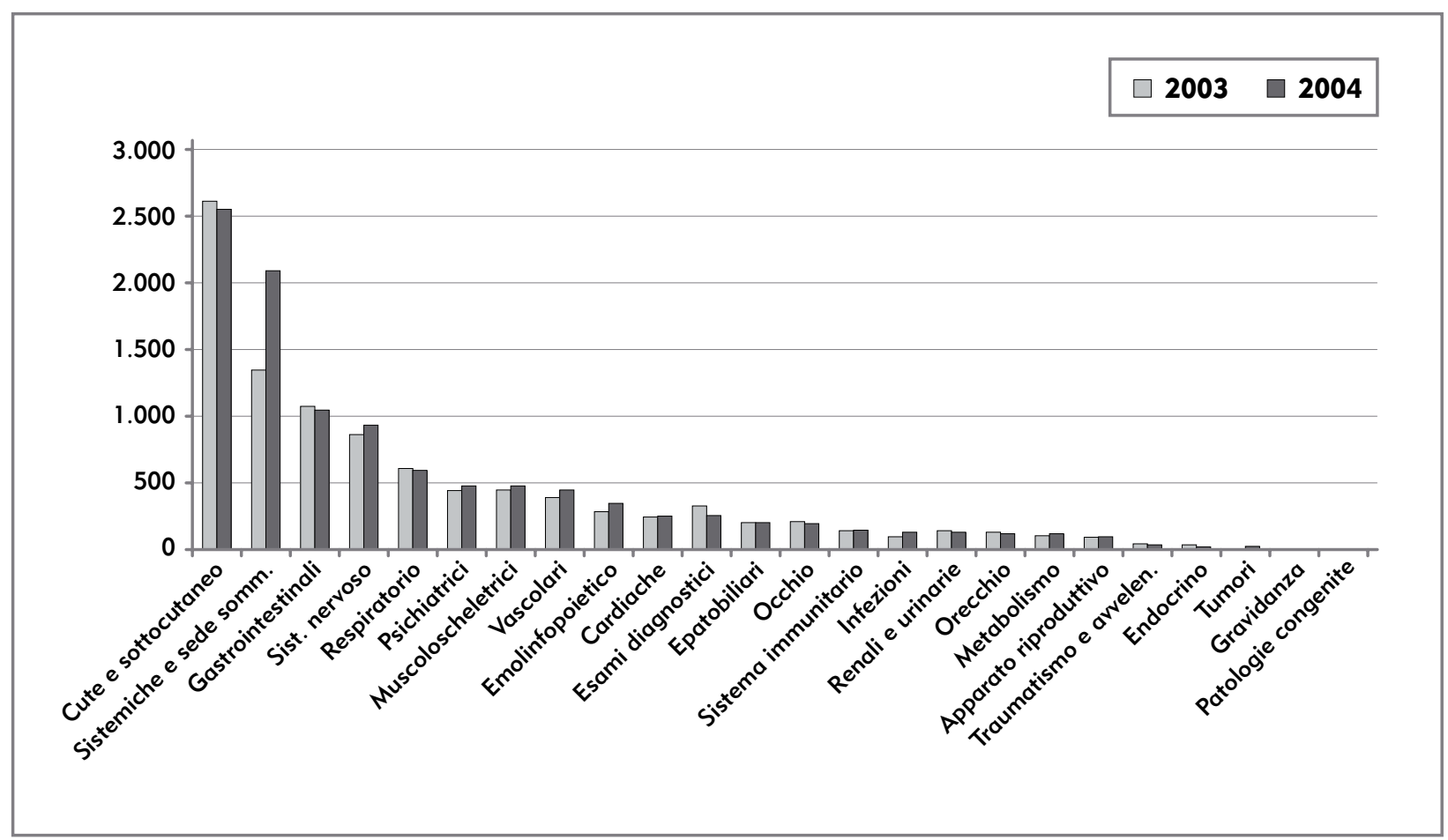

Figura 1. Reazioni avverse ai farmaci distinte per apparato

crociata con farmaci (cefalosporine) dannosi per la paziente, avrebbe potuto suscitare una reazione avversa, come poi nella realtà è sato, allora gli elementi di giustificazione della condotta del Sanitario si affievoliscono in modo apprezzabile. Ad onor del vero, la cute è l'organo del corpo che più di ogni altro costituisce sede di reazioni avverse da farmaci, come è comunemente noto e come si evince dalla Figura 1, che riassume un recente studio del ministero della Salute [17]; la cute è infatti sede del SALT (Skin Associated Lymphoid Tissues) e quindi frequentemente sede di risposte variabili agli stimoli esogeni, talvolta in modo esagerato.

D'altro canto, a difesa del Sanitario è da considerare che nella pratica ambulatoriale è difficile pensare che una reazione alimentare ai prodotti caseari o altro, oltretutto non meglio definita dalla paziente, possa invece costituire un elemento indiretto di allergia ad antibiotici, come in effetti eccezionalmente può verificarsi in caso di contaminazione o trattamento delle carni o del latte vaccino con antibiotici penicillinici [18-20] o con classi di antibiotici con i primi accomunati da reattività crociata.

\section{CONSIDERAZIONI SUL GIUDIZIO DI RESPONSABILITÀ}

Il giudizio di responsabilità professionale risente della prevedibilità dell'evento dannoso, della violazione dell'obbligo di informazione al paziente, dell'omissione del consenso consapevole di quest'ultimo all'atto medico, a maggior ragione se offlabel, e infine della specifica prestazione d'opera sanitaria, qualora essa sia causa efficiente del danno allegato.

Lo studio delle ADR è imprescindibile ai fini della definizione di eventuali comportamenti sanitari colposi, in quanto quelle di tipo A sono dose-dipendenti e prevedibili in funzione delle caratteristiche del farmaco, mentre quelle di tipo B sono dose-indipendenti e imprevedibili [21-23].

La maggior parte delle reazioni avverse a farmaci è dovuta al meccanismo d'azione stesso della molecola, ossia sono di tipo A; in tal caso il medico avendone conoscenza preventiva, per non esserne ritenuto responsabile dovrebbe mettere in atto tutti gli accorgimenti opportuni per evitarle, prevenendo così il danno alla persona. La grave reazione descritta nel caso in esame rientra invece tra le ADR di tipo B: esse, essendo per definizione imprevedibili, non sono al medico imputabili, secondo costante dottrina, copiosa giurisprudenza sia di merito che di legittimità e soprattutto secondo quanto previsto dall'ordinamento normativo giuridico [24], a patto che costituiscano prima manifestazione e a patto che l'anamnesi, ben condotta, sia stata negativa per episodi precedenti, escludendo in tal senso colpe eventuali per omissioni del paziente o dei suoi familiari.

La tematica dell'adeguata informazione del medico al suo assistito in merito al programma diagnostico-terapeutico a lui proposto è di scottante attualità, in quanto oggigiorno i giudizi di responsabilità professionale stanno interessando sempre più tale dovere del medico; recentissima giurisprudenza ha fatto estrema chiarezza sull'argomento, elevando il dovere etico-morale di informazione medica a requisito giuridico obbliga- 
torio, con precise conseguenze medico-legali in caso di sua omissione [25].

Nel caso specifico, il medico avrebbe dovuto prospettare in modo esaustivo alla sua assistita i rischi comuni e meno comuni della terapia proposta e le alternative praticabili al posto del piano terapeutico primario [26,27]. Dall'esame della documentazione sanitaria agli atti non è stato possibile appurare se ciò sia avvenuto; spetta quindi al Sanitario allegare ulteriori elementi per provare che l'informazione è stata ben effettuata, in base anche alle capacità cognitive della sua assistita, e che il consenso è stato richiesto e ottenuto.

A maggior ragione, tale dimostrazione è necessaria in virtù della terapia off-label prescritta ed effettuata; oltretutto il consenso informato rappresenta occasione di riduzione del contenzioso in quanto mira alla duplice tutela della salute del paziente e della professione e professionalità del Sanitario, costituendo a tutti gli effetti, anche in modalità verbale, una prova diretta di operato accorto e diligente (art. 1176 del Codice civile).

Dal punto di vista medico-giuridico, purtroppo, la condotta prescrittiva del Sanitario, non trova piena giustificazione neanche richiamando l'art. 23 della Legge 94/98, che legittima l'uso off-label dei farmaci non autorizzati per indicazioni e vie di somministrazioni non previste nell'allegata scheda tecnica. Il medico, infatti si è avvalso dell'uso off-label del farmaco, il quale, secondo scheda tecnica, può essere utilizzato solo ed esclusivamente per via iniettiva. La giustificazione della somministrazione per via locale sarebbe stata congrua in assenza di efficaci alternative terapeutiche verso un agente microbico sensibile alla sola penicillina. Poiché non è stata effettuata alcuna diagnosi microbiologica specifica, di conseguenza, sarebbe stato più opportuno somministrare, anche in modalità terapeutica ex adiuvantibus, qualsiasi medicamento topico battericida o batteriostatico a largo spettro d'azione indicato per la cura di lesioni infette, purché fornito di autorizzazione specifica per l'uso esterno e soprattutto non rischioso per la paziente; quindi ricorrendo valide alternative praticabili, nel caso di specie è venuto meno uno dei fondamentali parametri "giustificatori" dell'uso off-label dei medicamenti.

In aggiunta non ricorreva lo stato di necessità e urgenza, che avrebbe legalmente giustificato qualsiasi sensato intervento finalizzato a salvare una vita umana; a tal proposito si riportano in Tabella II i criteri permissivi della terapia off-label, previsti dall'art. 3 del D.L. 17 febbraio 1998, n. 23, convertito, con modificazioni, nella Legge 8 aprile 1998 , n. 94 pubblicata su Gazzetta Ufficiale n. 86 del 14 aprile 1998, il cui oggetto è l'effetto benefico di un farmaco, non autorizzato per la specifica indicazione e via di somministrazione, in riferimen-
1. Farmaco prodotto industrialmente e registrato nella Farmacopea Ufficiale

2. Inesistenza di altri farmaci registrati per quella indicazione

3. Esistenza di lavori qualificati apparsi su pubblicazioni scientifiche validate e accreditate in campo internazionale

4. Valutazione del rapporto rischio-beneficio

5. Necessità e indifferibilità terapeutica per le condizioni cliniche (stato di necessità)

6. Uso "compassionevole" del farmaco, specie in ambito oncologico

Tabella II. Criteri permissivi di prescrizione in modalità off-label

to ad una patologia non altrimenti curabile, argomento ripreso dalla Sentenza del 21 luglio 2000 del tribunale di Milano, sezione X penale.

\section{CONCLUSIONI}

L'interessante caso pratico riportato è opportuna occasione di riflessioni cliniche, medico-legali e giuridiche in merito a un'accusa di presunta responsabilità professionale, che merita di essere dimostrata con "apprezzabili probabilità" in ambito civile e "oltre ogni ragionevole dubbio" in ambito penale [28], ricorrenza quest'ultima da non prendere più in considerazione, nel caso specifico, per la rinuncia della parte lesa a procedere. Rimane il procedimento civile per la richiesta di risarcimento per danno biologico temporaneo, danni patrimoniali e morali allegati dalla parte lesa. Escludendo a priori il comportamento doloso del Sanitario, permane la discussione su quello colposo, solo in parte affievolito dalle giustificazioni da egli addotte in dibattimento. Di certo l'informazione e l'anamnesi sono state carenti e i requisiti permissivi prescrittivi della terapia off-label non a pieno rispettati. A ciò potrebbe essere aggiunta la critica che, almeno durante la prima somministrazione del farmaco, sarebbe stata necessaria la presenza del Sanitario, per ogni pur remota evenienza, poi nella realtà verificatisi, stante il dubbio sul rischio allergologico della paziente. Di contro la scelta del medico di non somministrare l'antibiotico per via generale ha probabilmente evitato un esito più grave e ciò deve essere tenuto in debito conto nel giudizio finale, che si augura non mortificare la persona, la professione e la professionalità del Sanitario e nello stesso tempo permettere il giusto risarcimento alla paziente, con differimento del relativo onere a carico della compagnia di assicurazione, in caso di sussistenza della tutela assicurativa a favore del Sanitario. 


\section{BIBLIOGRAFIA}

1. Staffa C. Qualità dei metodi analitici per il dosaggio delle IgE specifiche. Atti $72^{\circ}$ corso CEFAR di allergologia, Bologna, 18-19 dicembre 1996

2. Salkind AR, Cuddy PG, Foxworth JW. Is this patient allergic to penicillin? An evidence- based analysis of the likelihood of penicillin allergy. JAMA 2001; 285: 2498-505

3. Etter RL, Merryman G. Anaphylactic shock and death due to penicillin; report of a case. Ann Allergy 1954; 12(4): 453-4

4. Icasiano CB. Almost fatal penicillin anaphylactic-like shock reaction; report of a case at V. Luna General Hospital.J Philipp Med Assoc 1953; 29(9): 472-6

5. Eisenstadter D, Hussar AE. Severe anaphylactic reaction from oral penicillin. Am Pract Dig Treat 1954; 5(10): 783-4

6. Solensky R. Hypersensitivity rections to ß-lactam antibiotic. Clin Rev Allergy Immunol 2003; 4: 1-19

7. Nistrom CA. Anaphylactic reaction: a violent reaction to topical penicillin.J Kans Med Soc 1958; 59: 7

8. Weiss LR. Anaphylactic: report of a case. J Allergy 1953; 24: 407-10

9. Eedy DJ. Mupirocin allergy in the setting of venous ulceration. Contact Dermatitis 1995; 32: 240-1

10. Zappi EG, Brancaccio RR. Allergic contact dermatitis from mupirocin ointment. J Am Acad Dermatol 1997; 36: 266-7

11. Sauer S. Allergia alla bacitracina. Disponibile su www.lapelle.it/allergie/allergia_alla_bacitracina.htm

12. Sprung J, Schedewie HK, Zampine JP. Intraoperative anaphylactic shock after bacitracin irrigation. Anesth Analg 1990; 71: 4303

13. Errigo E. Malattie allergiche. Roma: Lombardo Editore, 1999. Terza Edizione, volume 1-20: 469-81

14. Cirfera V, Prete C. Medici accusati ingiustamente: effetto boomerang. Disponibile su www.dermatologialegale.it/index. php?pres $=33$

15. Muller U et al. Emergency treatment of allergic reactions to Hymnoptera sting. A position paper. Cli Exp Allergy 1991; 21: 281-8

16. ENDA, European Network of Drug Allergy. EAACI, European Academy off Allergy and Clinical Immunology

17. Le reazioni avverse ai farmaci nel 2004. BIF 2005; 5-6: 219-21

18. Ricci A, Vio D, Zavagnin P et al. Monitoraggio dell'antibioticoresistenza in batteri zoonotici e commensali isolati da bovini al momento della macellazione. Atti della Società Italiana di Buiatria 2003; 35: 59-66

19. Van den Bogaard AE, Stobberingh EE. Epidemiology of resistance to antibiotics. Links between animals and humans. Int J Antimicrobial Agents 2000; 14: 327-35

20. Commissione CE, dir. 97/72/CE del 15 dicembre 1997, in materia di "Additivi nell'alimentazione degli animali” in G.U. n. 351 del 23 dicembre 1997

21. Rawlins MD. Clinical pharmacology: adverse reactions to drugs. BMJ 1981; 282: 974-6

22. Rawlins MD, Thompson JW. Pathogenesis of adverse drug reactions. In: Davies DM. Textbook of adverse drug reactions. Oxford: Oxford University Press, 1977

23. Aronson JK e Ferner RE. Joining the DoTS: new approach to classifying adverse drug reactions. BMJ 2003; 327: 1222-5

24. Codice civile, art. 1225, in materia di "Prevedibilità del danno"

25. Cassazione civile, 14 marzo 2006, sentenza n. 5444 e Cassazione civile, sez. III, 19 ottobre 2006, sentenza n. 22390

26. Cirfera V. Il consenso informato in dermatologia. Atti del XV Congresso Nazionale AIDA, Associazione Italiana Dermatologi Ambulatoriali, Roma, 16-20 maggio 2006

27. Vinci P. La responsabilità professionale del medico-legale: il consenso informato. Le giornate doriche di medicina legale assicurativa, Ancona, 15 giugno 2006

28. Corte di Cassazione, 15 novembre 2002, sentenza n. 38334

\section{CORRESPONDING AUTHOR}

Dott. Valerio Cirfera, e-mail: info@dermatologialegale.it 